\title{
Shock-Wave-Induced Jetting of Micron-Size Bubbles
}

\author{
C. D. Ohl* and R. Ikink \\ Department of Applied Physics, Physics of Fluids, TU Twente, Postbus 217, 7500 AE Enschede, The Netherlands
} (Received 24 September 2002; revised manuscript received 18 February 2003; published 30 May 2003)

\begin{abstract}
Free gas bubbles in water with radii between 7 and $55 \mu \mathrm{m}$ subjected to a shock wave exhibit a liquid jetting phenomenon with the jet pointing in the direction of the propagating shock wave. With increasing bubble radius, the length of the jet tip increases and a lower estimate of the averaged jet velocity increases linearly from 20 to $150 \mathrm{~m} / \mathrm{s}$. At a later stage, the jet breaks up and releases micronsize bubbles. In the course of shock wave permeabilization and transfection of biological cells, this observation suggests a microinjection mechanism when the cells are near bubbles exposed to a shock wave.
\end{abstract}

DOI: $10.1103 /$ PhysRevLett.90.214502

Introduction.-This Letter focuses on the forced compression of a spherical gas bubble in the size range from 7 to $55 \mu \mathrm{m}$ in water by a shock wave. This research is stimulated by the question: Which physical mechanism might lead to a reparable opening of a cell membrane allowing the transfection of cells with large molecules which are normally blocked? For example, Delius and Adams [1] showed that shock waves from an extracorporeal lithotripter (ESWL) are able to transfer ribosomeinactivating proteins (gelonin and saporin) in vitro and in vivo into cells having no receptors for these proteins at the cell membrane.

As will be shown in this Letter, bubbles of the size of a few microns, even at moderate pressure amplitudes of $10 \mathrm{MPa}$, form a liquid jet in the direction of the propagating shock wave, which is in contrast to the findings of the numerical work of Ding and Gracewski [2]. There, shocks waves of $30 \mathrm{MPa}$ did not cause jetting.

Generally, the liquid jets form when a pressure gradient is present during the shrinkage (collapse) of a bubble. Two widely investigated examples are the collapse of a cavitation bubble near a rigid boundary (see, e.g., [3-5]), and the forced compression of gas bubbles by a passing shock wave [6-8].

When gas bubbles attached to foils are shocked, they exhibit jet speeds ranging from 400 to $790 \mathrm{~m} / \mathrm{s}$ for bubble radii between 0.15 and $0.9 \mathrm{~mm}$ [9]. The jet formation and its penetration into viscoelastic materials has recently motivated numerous works investigating the collapse of gas bubbles attached to tissue and tissue-mimicking materials when being subjected to a shock wave.

Previous experimental studies of shock compressed bubbles in liquids differ from the one presented here: First, the size of the gas bubble is considerably smaller and, second, the bubbles are not attached to a rigid or semirigid surface but rise freely. Thus, the influence of the shock wave only on the jet formation is demonstrated.

Experimental apparatus and results.-The experimental setup, depicted in Fig. 1, consists of the shock wave source, a bubble generator, the imaging and illumination devices, and digital delay lines. Shock waves are gener-
PACS numbers: 47.55.Bx

ated by a focused piezoelectric source; it is a slightly modified commercial extracorporeal lithotripter Piezolith 3000 (Richard Wolf GmbH, Knittlingen, Germany). The diameter of the shock wave source is $300 \mathrm{~mm}$ and the focusing angle $94^{\circ}$. The axis of the source is placed at an angle of $45^{\circ}$ to the horizontal at the bottom of a stainless steel container with glass windows on all four sides. The container is filled with degassed water $\left(3.3 \mathrm{mg} / 1 \mathrm{O}_{2}\right.$ concentration) at room temperature.

The pictures are taken with a sensitive slow scan CCD (charged coupled device) camera (Imager 3S with $1280 \times 1024$ pixels and $9 \mu \mathrm{m}$ pixel size, LaVision $\mathrm{GmbH}$, Germany) equipped with a long distance microscope (K2, Infinity, U.S.A.). The microscope operates from a working distance of $45 \mathrm{~mm}$ giving a maximum resolution of $0.85 \mu \mathrm{m}$ per pixel; however, in some experiments, the resolution was decreased by a factor of 2 or 4 by binning of pixels to enhance the signal-to-noise ratio. The CCD camera is operated in a double-frame mode, which allows one to take two images in rapid succession before they are transferred to a computer. Both frames are strobe illuminated with a LED (light emitting diode) driven either by a homebuilt current amplifier for exposure times down to $1.6 \mu \mathrm{s}$ or by a high voltage pulser (Panametrics, U.S.A.) to obtain shorter flashes of $150 \mathrm{~ns}$

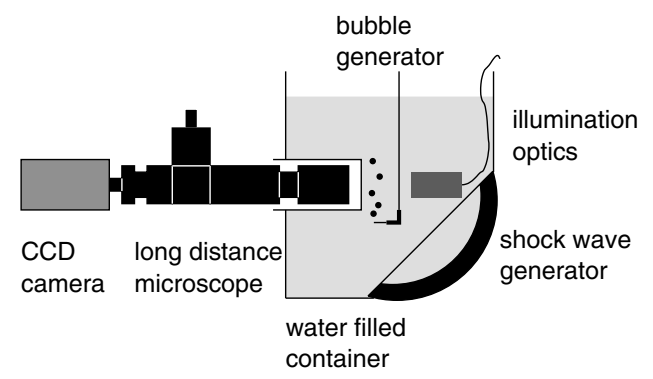

FIG. 1. Sketch of the experimental setup: The microscope, embedded partly in a cylindrical glass housing, is operated at a working distance of $45 \mathrm{~mm}$ from the focus of the shock wave generator. The bubble generator is positioned with an $x-y-z$ translation stage. 
duration. The light of the LED is coupled into a plastic fiber and brought to the sealed and submerged illumination optics placed in the container. All devices are triggered from digital delay generators.

Figure 2 displays a typical pressure recording taken with a calibrated PVDF (polyvinylidene fluoride) needle hydrophone (Dr. Pecha, University of Stuttgart) for a discharge voltage set to $5 \mathrm{kV}$. The wave consists of an overpressure cycle reaching an amplitude of $21 \mathrm{MPa}$ and lasting approximately $1 \mu \mathrm{s}$ followed by a pressure drop to $-3 \mathrm{MPa}$. The limited bandwidth of the hydrophone prevents one from resolving the rise time of the shock front accurately, which has the consequence that some uncertainty exists for the peak amplitude of the shock.

The inset of Fig. 2 displays on a larger time scale the pressure waves emitted from cavitation bubbles generated at the tip of the hydrophone which collapse for the first time $210 \mu$ s after the shock passage.

Swarms (1 to 10) of gas bubbles are generated approximately $3 \mathrm{~mm}$ below the focus of the shock wave generator with pulsed electrolysis. For an experimental run, the LED strobe is triggered twice, just before the shock enters the focal area, and as the shock passes through it. Figure 3 presents a typical frame sequence with an exposure time of $1.6 \mu \mathrm{s}$ (full width at half maximum) and $2.5 \mu \mathrm{s}$ interframe time. Here, it takes about $1.1 \mu$ s for the shock to travel under an angle of $45^{\circ}$ (see Fig. 1) to the horizontal from the bottom of the picture to the top towards the viewer. Clearly, all bubbles show a pronounced protrusion. In analyzing these images, we assume that the protrusion is in the direction of travel of the shock, i.e., at $45^{\circ}$ to the plane of view.

A likely sketch of the dynamics leading to the protrusion is given in Fig. 4 (left) and resembles what is seen in a magnified and motion-blurred view in Fig. 4 (right): The bubble translates upward, shrinks, and a protrusion pointing upward is formed. The sketch in Fig. 4 depicts the three stages. First, the bubble is hit by the shock at its

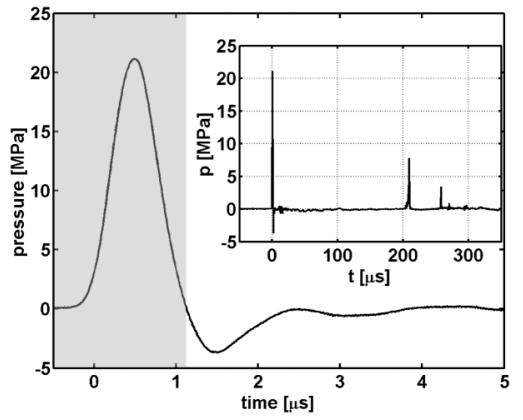

FIG. 2. Pressure versus time recorded with a PVDF needletype hydrophone. Experimental observations are limited before and to the time the overpressure acts (shaded gray). Note, the rise time of the positive peak is strongly overestimated due to the limited bandwidth of the hydrophone. Presumably it is around $10 \mathrm{~ns}$. lower wall. Because of a strong mismatch in acoustic impedances between water and gas, the shock becomes partially reflected and the part of the bubble wall parallel to the shock front gains an initial velocity approximately twice the particle velocity [7,11,12], which is here around $13 \mathrm{~m} / \mathrm{s}$. Second, the bubble is pushed forward due to the pressure radiation force $\mathbf{F}=-4 / 3 \pi R(t)^{3} \nabla P(t)$ and the overpressure causes the bubble to shrink leading with the conservation of impulse to an accelerating translational motion [13].

It is well known that translating and collapsing bubbles develop a jet in the direction of motion [13]. In this case, this effect is augmented by the velocity imparted by the shock wave, which varies along the bubble surface [12]. The jet flow becomes visible as a protrusion when it pushes the opposing bubble wall forming a gaseous layer around the liquid. Third, the protrusion becomes unstable and a tiny, presumably toroidal or liquid-filled, bubble remains at the jet tip, resembling the experiment of Ganan-Calvo and Gordillo et al. [14].

From the photograph, Fig. 4 (right), it is not possible to judge whether the bubble is still collapsing or already reexpanding; however, as the Rayleigh collapse time [9] $T_{C} \approx 0.9 R_{0} \sqrt{\rho / \Delta P}$, with $\rho$ the liquid density, is about $0.5 \mu \mathrm{s}, \Delta P=21 \mathrm{MPa}$, and $R_{0}=73 \mu \mathrm{m}$, and therefore short compared to the exposure time of $1.6 \mu \mathrm{s}$, the bubble is probably reexpanding. The center of the bubble translates by approximately one bubble radius; thus, the bubble center itself gains a velocity of $60 \mathrm{~m} / \mathrm{s}$ (again, note the perspective projection of $45^{\circ}$ ). Because of the strong curvature of the gaseous protrusion, the illumination, and the limited resolution of the imaging system, the liquid jet itself is not visualized in the photographs.

At a shorter exposure time of $150 \mathrm{~ns}$, the individual stages as sketched in Fig. 4 become clearer: Figure 5 depicts four bubbles rising upwards. The uppermost and largest bubble has formed the protrusion while, for the bubbles below, the gaseous layer visualizing the jet flow has already broken up, leaving two remains for each
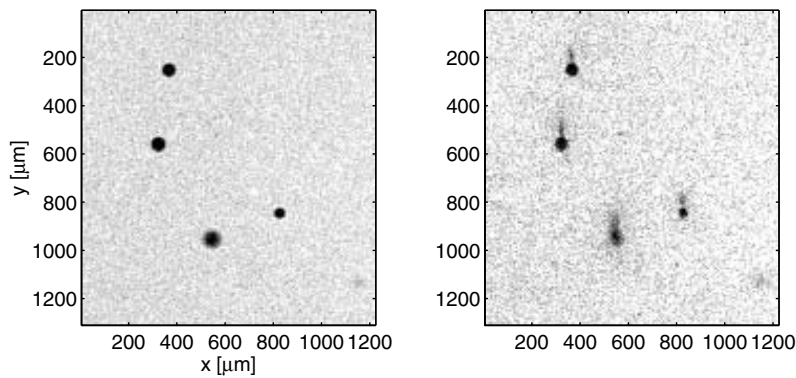

FIG. 3. The jetting of bubbles recorded with the two frame camera is displayed exemplarily. (left panel) The bubbles are shown before the shock enters the field of view from below. (right panel) $2.5 \mu \mathrm{s}$ later the bubbles have formed a protrusion in the direction of the shock wave passage. The exposure time is $1.6 \mu \mathrm{s}$. 
1)

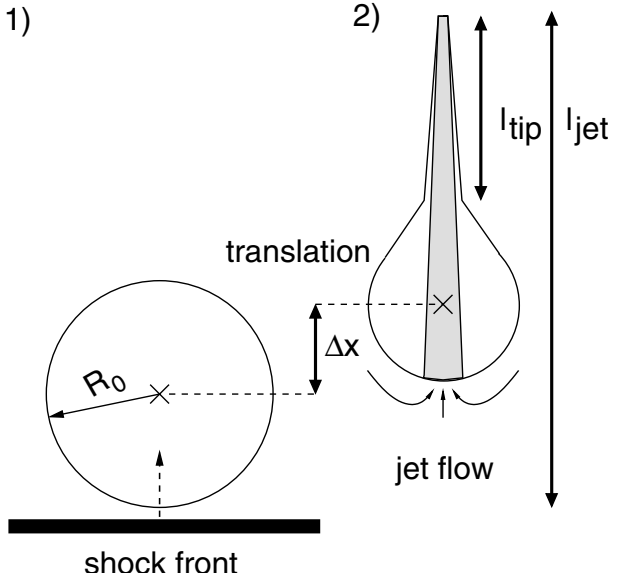

3)

(1)

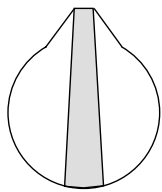

jet breakup

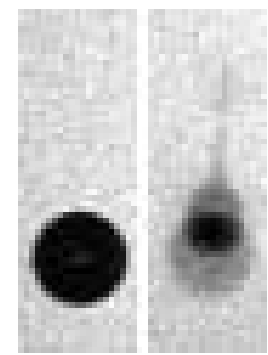

FIG. 4. (left panel) Sketch of the three stages in the course of liquid jetting: First the shock front impinges at the bottom of the bubble, then the bubble shrinks due to the overpressure, and its center is accelerated upwards. Liquid surrounding the bubble is focused on the rear and through the bubbles forming a protrusion (depicted gray) on the upper side. At last, the jet breaks up and a tiny presumably liquid filled bubble remains. (right panel) Photographs of a bubble before (left) and after the shock wave passage. The right image shows the bubble dynamics captured blurred (exposure time $1.6 \mu \mathrm{s}$ ) $2.5 \mu \mathrm{s}$ later than the left. The size of the individual frames is $(528 \times 182) \mu \mathrm{m}^{2}$. Please note that the shock wave is traveling under an angle of $45^{\circ}$ to the photographic view.

bubble, one from the main body and one much smaller one from the jet. Also, note that each bubble center is displaced.

Figure 6 shows measurements of the jet tip length $\left[l_{\text {tip }}\right.$ in Fig. 4 (left)], a lower bound of the jet velocity, and the bubble center displacement, $\Delta x$, for three low amplitudes between 11 and $21 \mathrm{MPa}$ and at a higher amplitude of $100 \mathrm{MPa}$ as a function of the initial bubble radius, $R_{0}$. For each pressure amplitude, 30-40 jetting bubbles were evaluated at largest magnification $(0.85 \mu \mathrm{m}$ per pixel). The liquid velocity $\left[v=l_{\text {jet }} / 1.6 \mu \mathrm{s}\right.$, see Fig. 4 (left)] was estimated from the fact that the liquid in contact with the bottom of the bubble has to travel into the tip of the jet

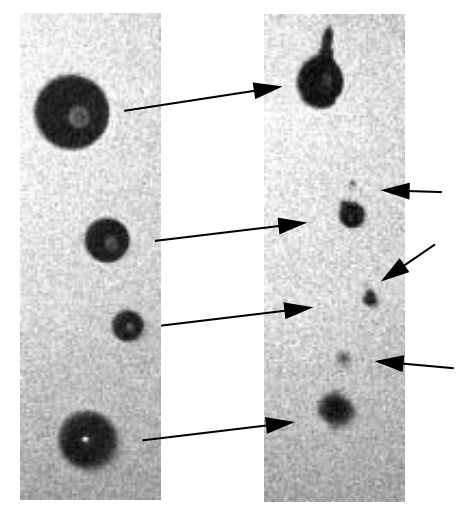

FIG. 5. Four bubbles $0.3 \mathrm{~ms}$ (left panel) before the shock wave arrives and $1.5 \mu \mathrm{s}$ (right panel) after the shock has entered from the bottom of the frame. The exposure time is $150 \mathrm{~ns}$ and the individual size of the frame is $(880 \times$ 250) $\mu \mathrm{m}^{2}$. The right pointing arrows indicate the center translation; the arrows pointing left depict the remaining bubble from the jet breakup. during the exposure time of $1.6 \mu \mathrm{s}$. Therefore, it is only a lower bound of the average flow velocity; the maximum velocity might be higher. All bubbles down to the smallest resolvable ones with a radius of $R_{0}=7 \mu \mathrm{m}$ exhibit a jet. The jet velocity, the center displacement, and the tip length scale approximately linearly with the bubble radius. The scatter of the tip length for larger bubbles might be caused by the fact that the tip of the jet traveled out of the depth of view and was therefore underestimated within the relatively large data scatter. We find no
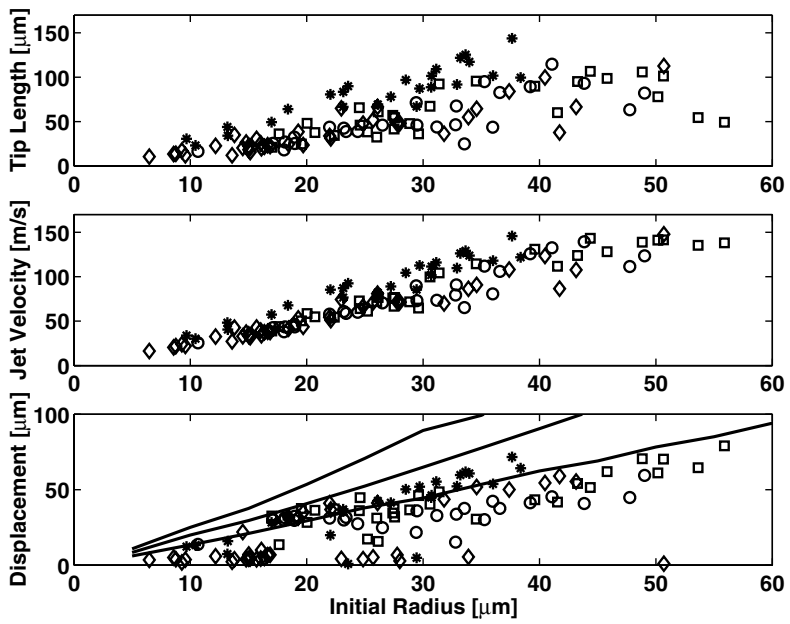

FIG. 6. The jet tip length, the displacement of the center, and the averaged velocity of the liquid jet is plotted versus the initial bubble radius. The symbols correspond to four different pressure amplitudes; $\bigcirc$ for $11 \mathrm{MPa}, \square$ for $14 \mathrm{MPa}, \diamond$ for $21 \mathrm{MPa}$, and * for $100 \mathrm{MPa}$, respectively. The solid lines depict the calculated center displacement for the three lowest pressure amplitudes. 
pronounced influence of the pressure amplitude on the data for the three lowest pressure amplitudes. One reason might be the small focal area of the shock wave generator having a full width at half maximum for the pressure amplitude of approximately $1.5 \mathrm{~mm}$. This is about the width of the frame. However, for the $100 \mathrm{MPa}$ case, the jet tip length and the liquid velocity increases by $50 \%$.

The displacement $\Delta x$ of the bubble due to the pressure rise is calculated with a simple model which only takes into account spherical bubble dynamics. The impinging pressure wave $p(x, t)$ is assumed to rise in a steplike fashion, $p(x, t)=\Delta P / 2 \tanh [\Omega(t-x / c)]$, with an amplitude $\Delta P$ and a rise time of $1 / \Omega$. The radial response is calculated with the Gilmore model [9], and the translational dynamics with a force balance model (for details, see $[15,16])$, leading to a system of four ordinary differential equations. The integration of the traveled distance is stopped at the time of minimum bubble volume. The rise time of the shock wave is assumed to be $10 \mathrm{~ns}$, thus $\Omega=10^{8} \mathrm{~s}^{-1}$. This is a typical value measured with membrane-type hydrophones [15]. An order of magnitude agreement between the calculated center displacement $(\Delta P=10,15$, and $20 \mathrm{MPa}$ plotted as solid lines in Fig. 6) and the measured values is obtained. The overestimation of the model can obviously be explained: During aspherical collapse, the jet within the bubbles travels faster than the radially shrinking main bubble; thus, the available duration for the acceleration is shortened, resulting in a shorter displacement [17].

Discussion and conclusions.-The results suggest that shock-collapsed bubbles might potentially act as microsyringes: Liquid around the bubble is accelerated into the tip of the jet, and if biological cells are in front the jet may inject liquid. The amount of liquid volume, $V_{j}$, within the jet protrusion can be related to the initial bubble radius, $R_{0}$, by assuming that the liquid jet has a cylindrical shape with cross sectional area $\pi R_{j}^{2}$ and length $l_{\text {tip }}$. The measurements suggest an approximate linear scaling between the length of the jet tip and the radius of the bubble $R_{0}, l_{\text {tip }} / R_{0} \approx 3$ [see Fig. 6 (top)] which holds down to the smallest bubbles detectable. A similar scaling for bubbles between 0.1 and $1 \mathrm{~mm}$ has been found by Kodama and Tomita [18]. Direct optical measurements of the diameter of the jet are difficult because of the strongly curved gaseous layer covering the jet. Nevertheless, an estimate can be obtained from pits produced by the jet on a gelatine surface as obtained by Kodama and Takayama [10]. Assuming these pits are caused by the liquid flow only (the momentum of the gas is negligible), the ratio between the pit radius $R_{j}$ and the bubble radius is $R_{j} / R_{0} \approx 0.1$. From the two ratios, the amount of liquid within the jet, $V_{j}$, is of the order of $V_{j} \approx$ $0.1 R_{0}^{3}$. For example, a $3 \mu \mathrm{m}$ bubble near a cell could inject at most a liquid volume of $V_{j} \approx 2.7 \times 10^{-15} 1$.
In this Letter, the jet formation from preexisting micron-size bubbles initiated by a shock front is demonstrated. It is found that small pressure amplitudes as compared to the protocol for stone fragmentation are sufficient. Further, the direction of the wave propagation controls the direction of the jet. This might open up an in vivo local drug delivery with micron-size drug-coated bubbles. However, for an application, future research is needed: It has to be demonstrated that cells can be temporarily permeabilized and transfected with these microjets. Additionally, the growth of the bubbles due to the shock wave trailing low pressure part might lead to unwanted side effects. Thus, a specially designed shock wave limiting the bubble growth might be advantageous.

The authors gratefully acknowledge the inspiring discussions with A. Prosperetti, D. Lohse, and M. Delius (Munich). This study has been supported by FOM (The Netherlands) under Grants No. 99MFS06 and No. 00PMT04.

*Electronic address: c.d.ohl@tn.utwente.nl

[1] M. Delius and G. Adams, Cancer Res. 59, 5227 (1999).

[2] Z. Ding and S. M. Gracewski, J. Fluid Mech. 309, 183 (1996).

[3] M. S. Plesset and R. B. Chapman, J. Fluid Mech. 47, 283 (1971).

[4] W. Lauterborn and H. Bolle, J. Fluid Mech. 72, 391 (1975).

[5] J. R. Blake, B. B. Tait, and G. Doherty, J. Fluid Mech. 170, 479 (1986).

[6] F. P. Bowden, F. R. S. Brunton, and J. H. Brunton, Proc. R. Soc. London A 236, 433 (1960).

[7] J. P. Dear, J. E. Field, and A. J. Walton, Nature (London) 332, 505 (1988).

[8] N. K. Bourne and J. E. Field, J. Fluid Mech. 244, 225 (1992).

[9] A. Philipp, M. Delius, C. Scheffczyk, A. Vogel, and W. Lauterborn, J. Acoust Soc. Am. 93, 2496 (1993).

[10] T. Kodama and K. Takayama, Ultrasound Med. Biol. 24, 723 (1998).

[11] G. Birkhoff, D. P. MacDougall, E. M. Pugh, and G. Taylor, J. Appl. Phys. 19, 563 (1948).

[12] M. P. Tulin, SIAM 725 (1969).

[13] T. B. Benjamin and A.T. Ellis, Philos. Trans. R. Soc. London, Ser. A 260, 221 (1966).

[14] A. M. Ganan-Calvo and J. M. Gordillo, Phys. Rev. Lett. 87, 274501 (2001).

[15] D. L. Sokolov, M. R. Bailey, and L. A. Crum, J. Acoust. Soc. Am. 110, 1685 (2001).

[16] C. D. Ohl, Phys. Fluids 14, 3512 (2002).

[17] Note, in some frames the lower border of the displaced bubble was blurred too much for an accurate reading of the displacement leading to the near zero displacement data points in Fig. 6.

[18] T. Kodama and Y. Tomita, Appl. Phys. B 70, 139 (2000). 